\title{
Erratum
}

\section{Erratum to: A polarisation modulation scheme for measuring vacuum magnetic birefringence with static fields}

\author{
G. Zavattini ${ }^{1,2, a}$, F. Della Valle ${ }^{3,4}$, A. Ejlli ${ }^{1,2}$, G. Ruoso ${ }^{5}$ \\ ${ }^{1}$ Dip. di Fisica e Scienze della Terra, Università di Ferrara, via G. Saragat 1, Edificio C, 44122 Ferrara, FE, Italy \\ ${ }^{2}$ INFN, Sez. di Ferrara, via G. Saragat 1, Edificio C, 44122 Ferrara, FE, Italy \\ ${ }^{3}$ Dip. di Fisica, Università di Trieste, via A. Valerio 2, 34127 Trieste, TS, Italy \\ ${ }^{4}$ INFN, Sez. di Trieste, via A. Valerio 2, 34127 Trieste, TS, Italy \\ ${ }^{5}$ INFN, Lab. Naz. di Legnaro, viale dell'Università 2, 35020 Legnaro, PD, Italy
}

(C) The Author(s) 2017. This article is an open access publication

Erratum to: Eur. Phys. J. C (2016) 76:294 https://doi.org/10.1140/epjc/s10052-016-4139-0

In the original article unfortunately we missed two typos in the equation for $I(\delta)$ at the top of the fourth page of the article. The equation should read

$$
\begin{aligned}
I(\delta) \approx & I_{0} \frac{T^{2}}{1-2 R \cos \delta+R^{2}} \\
& \times\left\{\eta(t)^{2}+\frac{2 \eta(t)\left(1-R^{2}\right)}{1-2 R \cos \delta+R^{2}}\left[\psi \sin \left(4 \phi(t)+4 \phi_{1}\right)\right.\right. \\
& \left.\left.+\alpha_{1} \sin \left(2 \phi(t)+2 \phi_{1}\right)+\alpha_{2} \sin \left(2 \phi(t)+4 \phi_{1}-2 \phi_{2}\right)\right]\right\} .
\end{aligned}
$$

The paper, the results and validity of the discussions are in no way altered by the typos in that they are all based on the correct equation.

Open Access This article is distributed under the terms of the Creative Commons Attribution 4.0 International License (http://creativecomm ons.org/licenses/by/4.0/), which permits unrestricted use, distribution, and reproduction in any medium, provided you give appropriate credit to the original author(s) and the source, provide a link to the Creative Commons license, and indicate if changes were made. Funded by SCOAP ${ }^{3}$.

The original article can be found online at https://doi.org/10.1140/ epjc/s10052-016-4139-0.

F. Della Valle: Now at: Dip. di Scienze Fisiche, della Terra e dell'Ambiente, Università di Siena, Via Roma 56, 53100 Siena, SI, Italy.

a e-mail: guido.zavattini@unife.it 